\title{
Weak localization of bismuth cluster-decorated graphene and its spin-orbit interaction
}

\author{
Jian-Lei Ge ${ }^{1}$, Tian-Ru $\mathrm{Wu}^{2}$, Ming Gao ${ }^{3}$, Zhan-Bin $\mathrm{Bai}^{1}$, Lu Cao ${ }^{1}$, \\ Xue-Feng Wang ${ }^{3}$, Yu-Yuan Qin ${ }^{1, \dagger}$, Feng-Qi Song ${ }^{1, \ddagger}$ \\ ${ }^{1}$ National Laboratory of Solid State Microstructures, Collaborative Innovation Center of Advanced Microstructures, \\ and Department of Physics, Nanjing University, Nanjing 210093, China \\ ${ }^{2}$ State Key Laboratory of Functional Materials for Informatics, Shanghai Institute of Microsystem and \\ Information Technology, Chinese Academy of Sciences, Shanghai 200050, China \\ ${ }^{3}$ School of Electronic Science and Engineering and National Laboratory of Solid State Microstructures, \\ Nanjing University, Nanjing 210093, China \\ Corresponding authors.E-mail: †qinyuyuan@nju.edu.cn, ${ }^{\ddagger}$ songfengqi@nju.edu.cn \\ Received December 22, 2016; accepted February 5, 2017
}

\begin{abstract}
Weak-localization (WL) measurements were performed in a Bi cluster-decorated graphene sheet. The charge concentration was kept constant, and the amplitude of the conductance correction was suppressed after the Bi-cluster deposition. Detailed WL data were obtained while the gate and temperature were changed. Using E. McCann's formula, the spin-relaxation time was extracted, which was found to increase with the elastic scattering time. This is attributed to the Elliott-Yafet spin relaxation and Kane-Mele type spin-orbit coupling (SOC). The SOC strength was enhanced to $2.64 \mathrm{meV}$ as a result of the first deposition. The coverage effect is discussed according to the measurement after the second deposition.
\end{abstract}

Keywords graphene, cluster deposition, weak localization, spin-orbit coupling

PACS numbers 36.40.-c, 72.80.Vp, 73.20.Fz

\section{Introduction}

Numerous efforts have been directed towards enhancing the intrinsic spin-orbit coupling (SOC) strength of graphene-based systems for potential applications in quantum spin Hall (QSH) devices, in which it is possible to realize dissipationless spin/charge transport by exploiting the strong topological protection arising from the anomalous electronic structures [1]. Several approaches have been suggested, such as utilizing the proximity effect in graphene/strong SOC material heterostructures [2-4], the hydrogenation and fluorination of graphene $[5,6]$, and depositing certain heavy adatoms on graphene [7-10]. Experimentally, angle-resolved photoemission spectroscopy did not reveal an SOC gap in epitaxial $\mathrm{Bi}_{2} \mathrm{Te}_{2} \mathrm{Se}$ on chemical vapor deposition (CVD)-

\footnotetext{
*Special Topic: Recent Progress on Quantum Transport in Nano and Mesoscopic Systems (Eds. Qing-Feng Sun, Zhen-Hua Qiao \& Xin-Qi Li).
}

grown graphene [11]. Scanning tunneling microscopy/ spectroscopy revealed a large spin-orbit splitting of the graphene Dirac states up to $80 \mathrm{meV}$ in CVD-grown graphene $/ \mathrm{Bi}_{2} \mathrm{Se}_{3}$ van der Waals heterostructures, but there were strong spatial fluctuations of the transferred SOC in graphene both in energy and crystal momentum [12] Graphene that is covalently bonded with small amounts of hydrogen atoms exhibits a large SOC enhancement, i.e., $2.5 \mathrm{meV}$, which is ascribed to the conversion of the bonding condition from $s p^{2}$ to $s p^{3}$ [13]. Graphene that adsorbs $\mathrm{Cu} / \mathrm{Au}$ adatom clusters has an SOC as high as $20 \mathrm{meV}$, giving rise to a giant spin Hall effect, because these $\mathrm{Cu} / \mathrm{Au}$ adatom clusters function as local spin-orbit scatterers and induce transverse spin currents with an enhanced skew-scattering contribution [14]. Recently, the interaction between the adatom clusters and the vacancy defects of graphene was predicted to induce a robust SOC $[15,16]$. The spin state originating from the interaction between adatoms and vacancies was observed via electron spectroscopy [17]. Herein, we report transport measurements of graphene before and 
after the deposition of Bi clusters. Analysis of the weaklocalization (WL) data at various back-gate voltages and temperatures revealed that the spin relaxation was dominated by the Elliott-Yafet mechanism and that the corresponding Kane-Mele type SOC was enhanced to 2.64 $\mathrm{meV}$ by the first deposition. The coverage effect is discussed according to the measurement after the second deposition.

\section{Experiments}

The graphene sample was grown via $\mathrm{CVD}$ on $\mathrm{Cu}$ foils. Subsequently, the $\mathrm{Cu}$ was etched in an $\mathrm{FeCl}_{3}$ solution and transferred onto a $\mathrm{Si} / \mathrm{SiO}_{2}$ substrate using the polymethyl methacrylate (PMMA)-assisted wet-transfer method [18]. Then, the PMMA was removed by acetone. The transferred samples were annealed in an $\mathrm{Ar} / \mathrm{H}_{2}$ (93:7) environment at $300{ }^{\circ} \mathrm{C}$ for $3 \mathrm{~h}$ to remove the residual organic contaminants. Electrode probes with 5 $\mathrm{nm}$ thick Ti and $40 \mathrm{~nm}$ thick Au films were made using the liftoff procedure and electron-beam evaporation, as shown in the inset of Fig. 1(a). The devices were annealed in an $\mathrm{Ar} / \mathrm{H}_{2}$ (93:7) environment at $300{ }^{\circ} \mathrm{C}$ for $3 \mathrm{~h}$ to remove the residual photoresist. The monolayer graphene was identified via Raman spectroscopy at room temperature. The transport measurement in each process was performed in a Janis refrigerator that had a base temperature of $5.5 \mathrm{~K}$ and was equipped with a $5 \mathrm{~T}$ superconductor magnet. The Keithley 6221 alternating- and directcurrent source and a Keithley $2182 \mathrm{~A}$ nanovoltmeter were utilized to measure the source current and voltage. The excitation current was $1 \mu \mathrm{A}$. The back-gate voltage was supplied by a Keilthley 2400 sourcemeter. The beam of the $\mathrm{Bi}$ clusters was generated in a gas-aggregation cluster source $[19,20]$. The surface morphology of the Bi cluster-decorated graphene was characterized via atomic force microscopy (AFM) (Asylum Research Cypher S).

\section{Results and discussion}

\subsection{Influence of Bi clusters on transport behavior of graphene}

Figure 1(a) shows the Raman spectrum of the pristine graphene. The $2 \mathrm{D} / \mathrm{G}$ peak ratio is 2.07 . According to the Lorentz fitting to the Raman data, the full width at half maximum of the $2 \mathrm{D}$ peak was $29.9 \mathrm{~cm}^{-1}$. This confirms the presence of monolayer graphene. The defects in this graphene are confirmed by the large D/G peak ratio of 0.65 . Figure 1 (b) shows the temperature dependence of the longitudinal resistance $(R-T)$ of the graphene device before $(\mathrm{C} 0)$ and after the first Bi-cluster deposition (C1). The overall metallic behavior of the $R-T$ curves is observed, which agrees with a previous report [21]. Figure 1(d) shows the relationship between the longitudinal resistance and the back-gate voltage $\left(R-V_{g}\right)$ before and after the Bi-cluster deposition. The pristine and Bi cluster-decorated graphene were p-doped. Using Hall measurements, we obtained the charge density as $n=1 /\left(q R_{H}\right)$, where $q$ is the electronic charge, and $R_{H}$ is the Hall coefficient obtained by fitting $R_{x y} \sim B(T)$ with the linear curve at a low magnetic field $( \pm 1 \mathrm{~T})$. As shown in Fig. 1(e), the decoration of Bi clusters reduced the hole-doping level of the graphene. The carrier mobility of the device was obtained as $\mu=\sigma /(n q)$, where $\sigma$ is the conductivity, as shown in Fig. 1(f). After the Bi-cluster deposition, the carrier mobility was reduced because the clusters contributed scattering centers, as reported in previous experiments [22-24]. The transport parameters of the graphene before and after the Bi-cluster deposition are detailed in Table 1.

\subsection{Influence of Bi clusters on WL of graphene}

WL is a phase-coherent backscattering effect resulting from the interference of a closed path with its reversed counterpart. The chiral carriers and nontrivial Berry phase [25] in the monolayer graphene lead to its intrinsic weak antilocalization (WAL). However, the interplay between the lattice, valley, and spin degrees of freedom, as well as the relative strength of different types of symmetry-breaking scattering, gives rise to the regimes of WL and WAL behavior [26-28]. At a low temperature, the WL may be sensitive to the presence and nature of the SOC. E. McCann developed a theory for analyzing the WL in graphene, considering the SOC $[26,27]$. According to this theory, both the Bychkov-Rashba SOC and the Kane-Mele SOC influence the WL. If BychkovRashba SOC is present, this tends to induce WAL in graphene, but if intrinsic Kane-Mele SOC prevails, the WL is suppressed $[29,30]$. The following formula is adapted from the low-field magnetoresistance formula proposed by E. McCann,

Table 1 Parameters for the device before $(\mathrm{C} 0)$ and after the first (C1) and second deposition of Bi clusters (C2). The resistance $R$ was measured at room temperature, whereas the charge concentration $n$, mobility $\mu$, elastic scattering length $l_{e}$, and diffusion coefficient $D$ were measured at $5.5 \mathrm{~K}$.

\begin{tabular}{cccccc}
\hline & $R(\Omega)$ & $n\left(10^{12} \mathrm{~cm}^{-2}\right)$ & $\mu\left(\mathrm{cm}^{2} \cdot \mathrm{V}^{-1} \cdot \mathrm{s}^{-1}\right)$ & $l_{e}(\mathrm{~nm})$ & $D\left(\mathrm{~cm}^{2} \cdot \mathrm{s}^{-1}\right)$ \\
\hline C0 & 320.7 & 4.0 & 1631.0 & 38.0 & 190.0 \\
C1 & 334.4 & 3.9 & 1256.5 & 25.2 & 126.1 \\
C2 & 880.9 & 3.5 & 396.3 & 9.9 & 49.4 \\
\hline
\end{tabular}




$$
\begin{aligned}
\Delta \sigma(B)= & -\frac{e^{2}}{2 \pi h}\left[F\left(\frac{B}{B_{\varphi}}\right)-2 F\left(\frac{B}{B_{\varphi}+B_{*}}\right)-F\left(\frac{B}{B_{\varphi}+2 B_{i}}\right)-2 F\left(\frac{B}{B_{\varphi}+B_{\mathrm{BR}}+B_{\mathrm{KM}}}\right)\right. \\
& +4 F\left(\frac{B}{B_{\varphi}+B_{*}+B_{\mathrm{BR}}+B_{\mathrm{KM}}}\right)+2 F\left(\frac{B}{B_{\varphi}+2 B_{i}+B_{\mathrm{BR}}+B_{\mathrm{KM}}}\right)-F\left(\frac{B}{B_{\varphi}+2 B_{\mathrm{BR}}}\right) \\
& \left.+2 F\left(\frac{B}{B_{\varphi}+B_{*}+2 B_{\mathrm{BR}}}\right)+F\left(\frac{B}{B_{\varphi}+2 B_{i}+2 B_{\mathrm{BR}}}\right)\right] .
\end{aligned}
$$

(a)

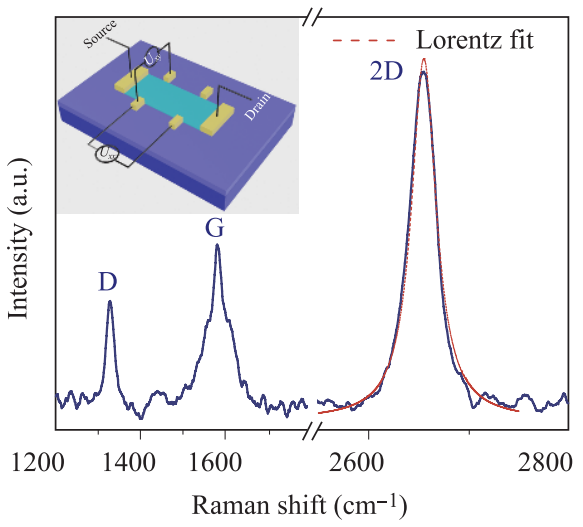

(c)

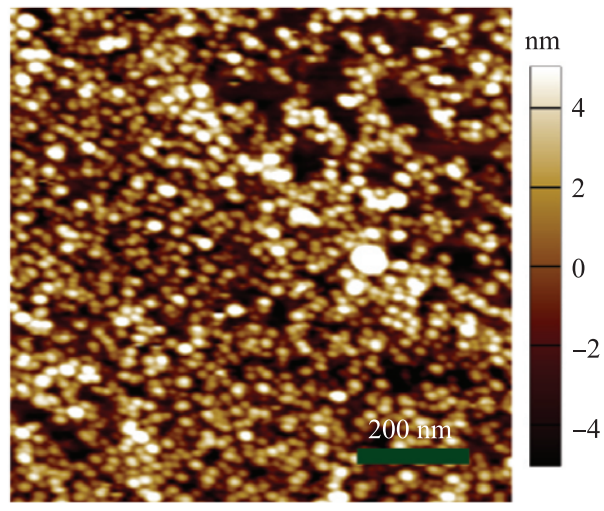

(e)

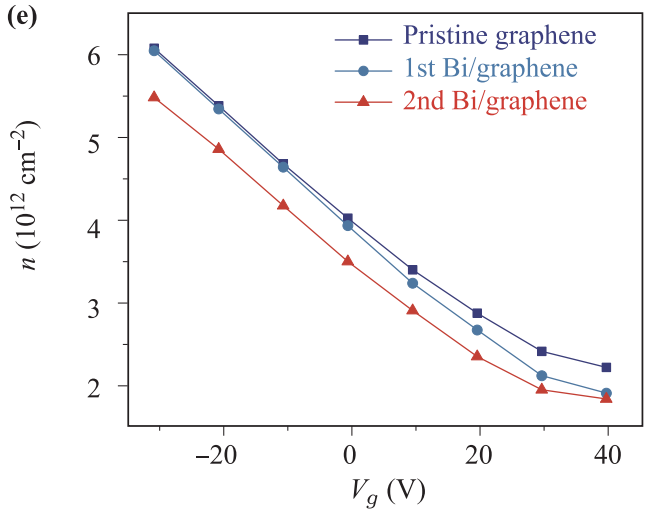

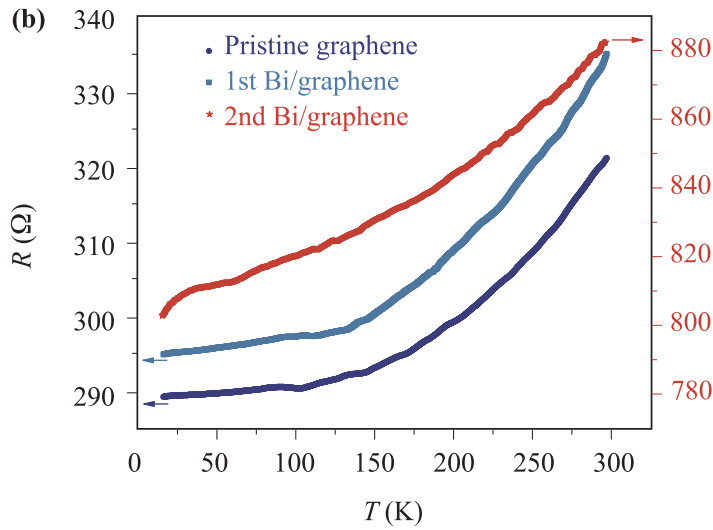
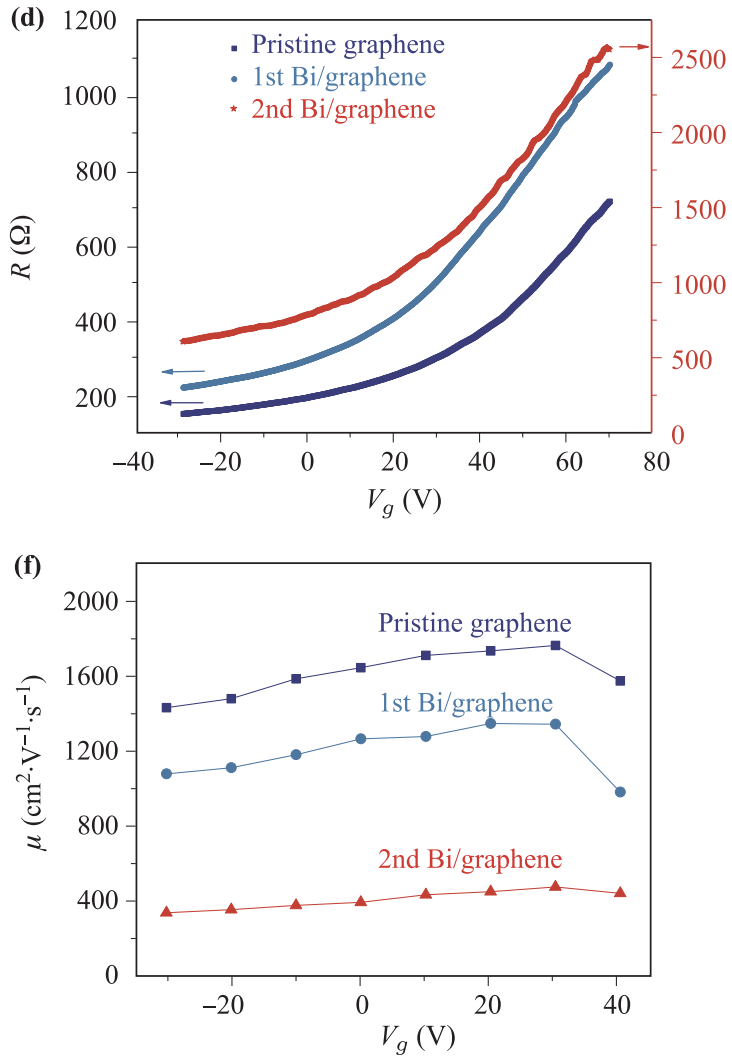

Fig. 1 Structural and transport characterization of the device. (a) Raman spectrum of pristine graphene and its schematic configuration. The red dashed line represents the Lorentz fitting. (b) Resistance with respect to the temperature for $\mathrm{C} 0$, $\mathrm{C} 1$, and $\mathrm{C} 2$. (c) AFM image of $\mathrm{C} 2$, where the clusters are approximately $7 \mathrm{~nm}$ in size. The scale bar represents $200 \mathrm{~nm}$. (d) Resistance with respect to the back-gate voltage $\left(V_{g}\right)$ for $\mathrm{C} 0, \mathrm{C} 1$, and $\mathrm{C} 2$. (e) Carrier density of $\mathrm{C} 0, \mathrm{C} 1$, and $\mathrm{C} 2$ plotted with respect to the back-gate voltage. (f) Mobility of $\mathrm{C} 0, \mathrm{C} 1$, and $\mathrm{C} 2$ plotted with respect to the back-gate voltage. 
Here, $F(z)=\ln z+\psi(1 / 2+1 / z), B_{\varphi, i, *, \mathrm{BR}, \mathrm{KM}}=$ $[\hbar c /(4 D e)] \tau_{\varphi, i, *, \mathrm{BR}, \mathrm{KM}}^{-1}, \psi$ is the digamma function, $D$ is the diffusion coefficient, $\tau_{\varphi}^{-1}$ is the inelastic dephasing rate, $\tau_{*}^{-1}$ is the intravalley symmetry-breaking scattering rate, $\tau_{i}^{-1}$ is the intervalley scattering rate, $\tau_{\mathrm{BR}}^{-1}$ is the extrinsic Bychkov-Rashba spin-relaxation rate, and $\tau_{\mathrm{KM}}^{-1}$ is the intrinsic Kane-Mele spin-relaxation rate. The Bychkov-Rashba SOC requires the breaking of the mirror symmetry in the graphene plane, which can destroy the QSH state [31-33]. On the other hand, the intrinsic Kane-Mele SOC satisfies a full invariant of the transformations of the point group, which can favor the existence of the QSH state. The Bychkov-Rashba SOC produces spin relaxation in the graphene through the D'yakonov-Perel mechanism, whereas the intrinsic Kane-Mele SOC causes spin relaxation in the graphene through the Elliott-Yafet mechanism [34]. The corresponding spin-relaxation rates are related to the SOC strength according to the equations $\tau_{\mathrm{BR}}^{-1}=2 \tau_{p} \Delta_{\mathrm{BR}}^{2} / \hbar^{2}$ and $\tau_{\mathrm{KM}}^{-1}=\tau_{p}^{-1}\left(\Delta_{\mathrm{KM}} / \varepsilon_{F}\right)^{2}$, where the elastic scattering rate is $\tau_{p}^{-1}=2 e^{2} v_{F} \sqrt{\pi n} /(h \sigma)$.

By tuning the back gate, we set the charge concentration of the graphene device as $4 \times 10^{12} \mathrm{~cm}^{-2}$ before and after the Bi-cluster deposition. Then, the low-field magnetoconductance (MC) data for pristine and Bi clusterdecorated graphene at the same charge concentration were compared, as shown in Figs. 2(a) and (b). The sharp dip of MC near $50 \mathrm{mT}$ and the decreasing amplitude with the increasing temperature indicates the WL effect. The $\mathrm{MC}$ data for pristine graphene at $50 \mathrm{~K}$ are not good, because the WL at this temperature was suppressed, and the noise was large. Obviously, with the Bi-cluster deposition, the WL at the same temperature decreased. This reveals the suppression of WL, indicating that the dominant SOC was the intrinsic Kane-Mele type. In a recent experiment, the electronic structure and spinorbit splitting in graphene/ $\mathrm{Ni}(111)$ intercalated with a high spin-orbit Bi material were studied via angle- and spin-resolved photoemission. The Rashba spin splitting of the Dirac cone is nearly zero. This is ascribed to the peculiar electronic structure of $\mathrm{Bi}$, which has a valence band with no d electronic states responsible for the onset of giant Rashba effect through electronic hybridization with the $\pi$ band of graphene [35]. Thus, it is reasonable for us to only consider the influence of the Kane-Mele SOC on the WL. We neglect the Bychkov-Rashba terms, reducing Eq. (1) to the following form:

$$
\begin{aligned}
\Delta \sigma(B)= & -\frac{e^{2}}{2 \pi h}\left[-2 F\left(\frac{B}{B_{\varphi}+B_{\mathrm{KM}}}\right)\right. \\
& +4 F\left(\frac{B}{B_{\varphi}+B_{*}+B_{\mathrm{KM}}}\right) \\
& \left.+2 F\left(\frac{B}{B_{\varphi}+2 B_{i}+B_{\mathrm{KM}}}\right)\right] .
\end{aligned}
$$
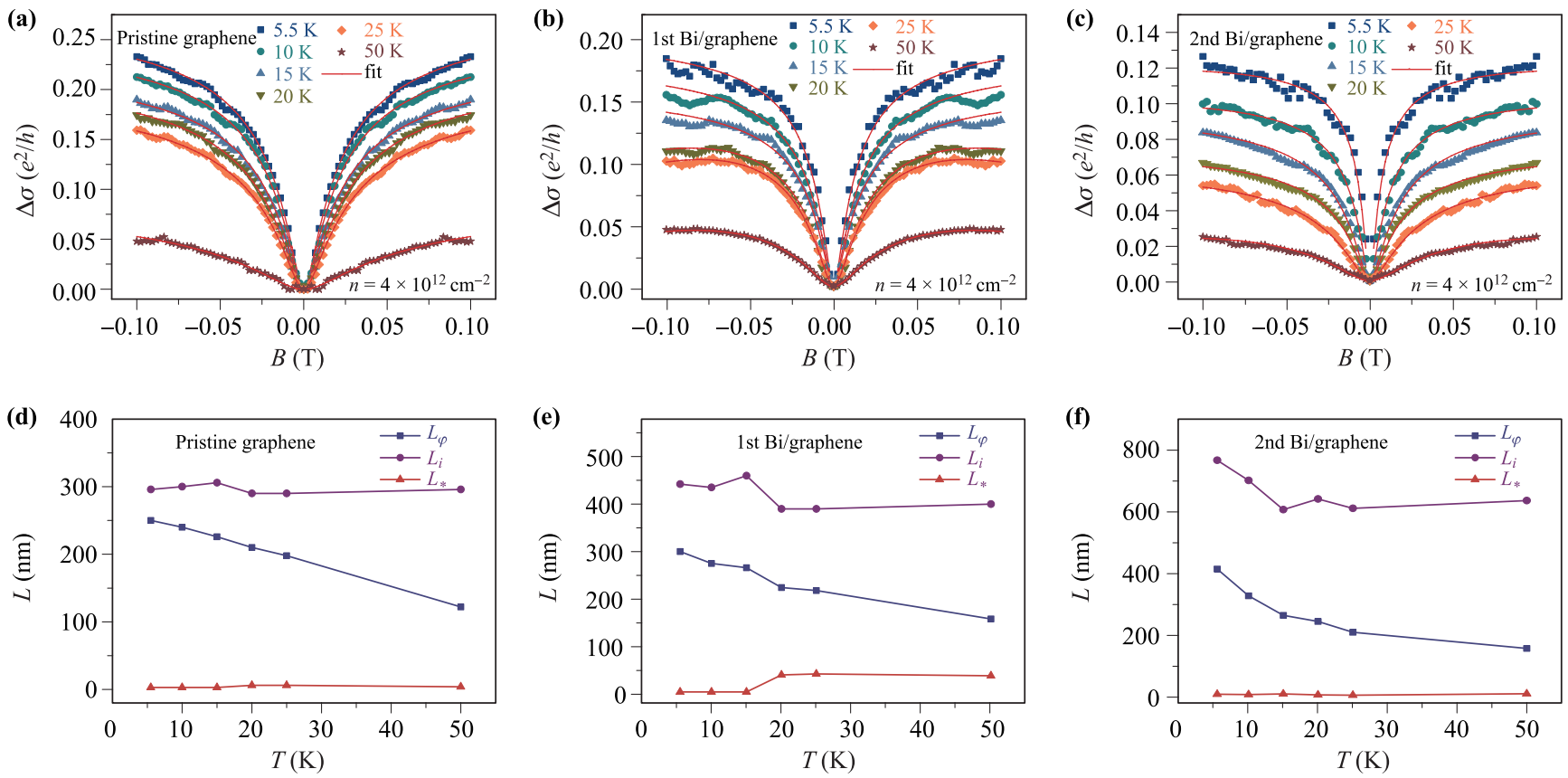

Fig. 2 WL analysis of the device at various temperatures and the same carrier density under three conditions. (a-c) WLs of $\mathrm{C} 0, \mathrm{C} 1$, and $\mathrm{C} 2$, respectively, with the same charge concentration at various temperatures. The conductance correction is suppressed by nearly $50 \%$ after the second Bi-cluster decoration. (d-f) $L_{\varphi}$ decreases as the temperature increases, because of electron-electron interactions. $L_{i}$ and $L_{*}$ do not change as the temperature increases. 
Fitting the WL data in Figs. 2(a) and (b) with this equation yields some parameters $-L_{\varphi, i, *}=\sqrt{D \tau_{\varphi, i, *}}$ - as shown in Figs. 2(d) and (e). The inelastic dephasing length $L_{\varphi}$ decreases with increasing temperature, which is attributed to electron-electron interactions. The intervalley scattering length $L_{i}$ and the intravalley scattering length $L_{*}$ do not change as the temperature increases, because the scattering centers remain stable during the measurement [36-38].

The WL data for the graphene device before and after the Bi-cluster deposition were compared systematically at various temperatures and back-gate voltages. The low-field MC of the device before and after Bi-cluster deposition at a series of back-gate voltages with a fixed temperature of $5.5 \mathrm{~K}$ is shown in Figs. 3(a) and (b). The changes of the characteristic lengths $L_{\varphi}, L_{i}$, and $L_{*}$ are shown in Figs. 3(d) and (e).

\subsection{Strength change in spin-orbit interaction caused by Bi-cluster deposition}

According to E. McCann's WL theory, determining the elastic scattering time and the spin-relaxation time at a series of charge concentrations results in a trustworthy fit to $\tau_{\mathrm{KM}}=\tau_{p}\left(\varepsilon_{F} / \Delta_{\mathrm{KM}}\right)^{2}$, which eventually gives the strength of the spin-orbit interaction. The extracted spin-relaxation times of the graphene before and after Bi-cluster deposition are plotted in Figs. 4(a) and (b). For pristine graphene, the spin-relaxation time reaches
1500 ps, which shows the superiority of graphene as a spin-transport material. The spin-relaxation time monotonically increases with the increase of the elastic scattering time, which is a characteristic of Elliott-Yafet spin relaxation. Therefore, we fit the data according to $\tau_{\mathrm{KM}}=\tau_{p}\left(\varepsilon_{F} / \Delta_{\mathrm{KM}}\right)^{2}$, where $\Delta_{\mathrm{KM}}$ is the strength parameter of the SOC causing the spin relaxation, and $\varepsilon_{F}$ is the chemical potential compared with the Dirac point. Good fitting is achieved at all the temperatures, as shown in Figs. 4(a) and (b). Thus, we determine the effective SOC strength of our graphene device before and after Bi-cluster deposition. The SOC strength increases with the temperature. The temperature-dependent SOC is attributed to the extrinsic SOC because it arises from the temperature-dependent electronic scattering caused by the impurity centers $[39,40]$. In the inset of Fig. 4(d), we plot the temperature-dependent gap with respect to the temperature. All the data points are close to the fitting curve, which can be extrapolated to zero temperature. These indicate the temperature-independent SOC strength, which is attributed to the intrinsic SOC. The temperature-independent SOC strengths of pristine and $\mathrm{Bi}$ cluster-decorated graphene are 0.91 and $2.64 \mathrm{meV}$, respectively.

\subsection{Coverage effect of Bi clusters}

In theories and experiments regarding adatoms on the graphene, the size of adsorbed heavy metal nanoclusters
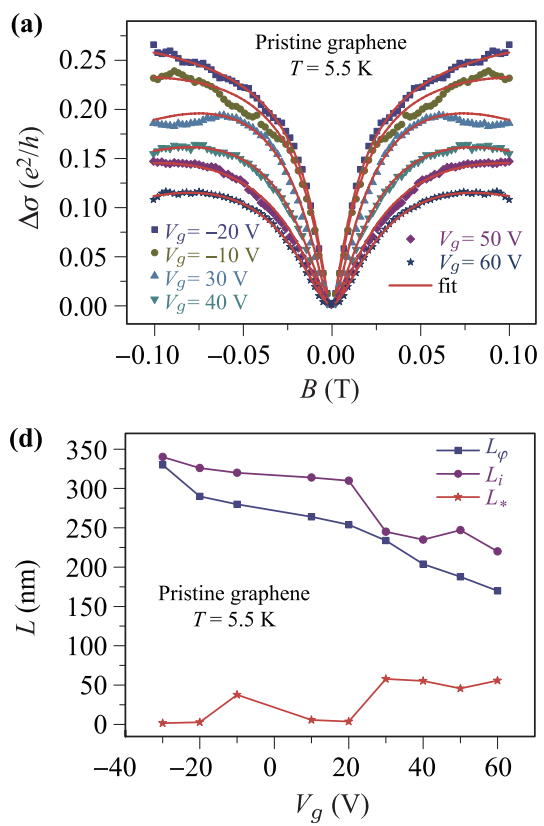
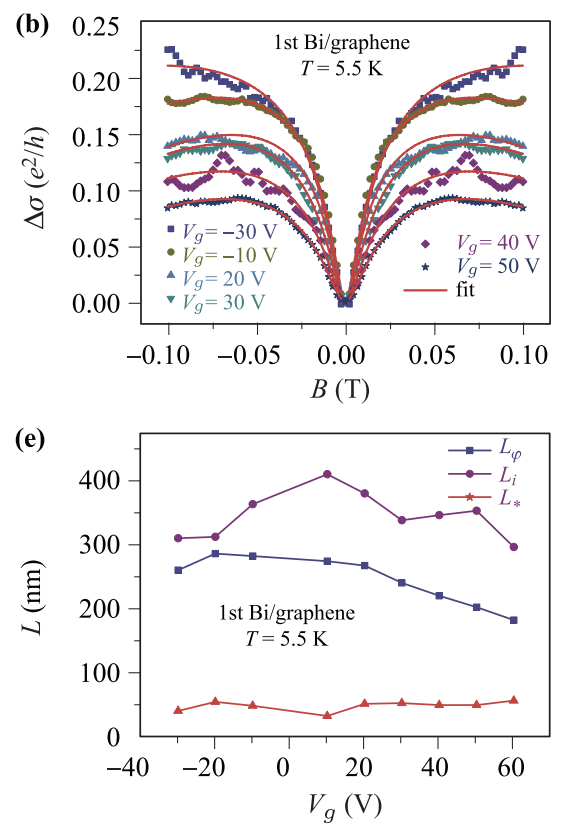
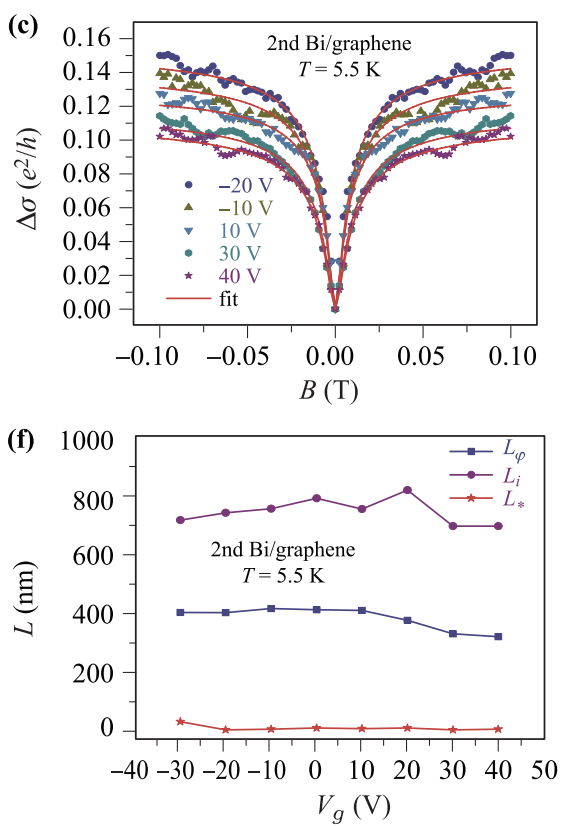

Fig. 3 WL analysis of the device with a varying gate voltage under three conditions. (a-c) WLs of C0, C1, and C2, respectively, with various gate voltages at $5.5 \mathrm{~K}$. The conductance correction is greatly suppressed when the Fermi level is moved near the Dirac point, because of the suppressed electronic screening. (d-f) Characteristic lengths obtained by fitting the WL data with respect to the back-gate voltage. 

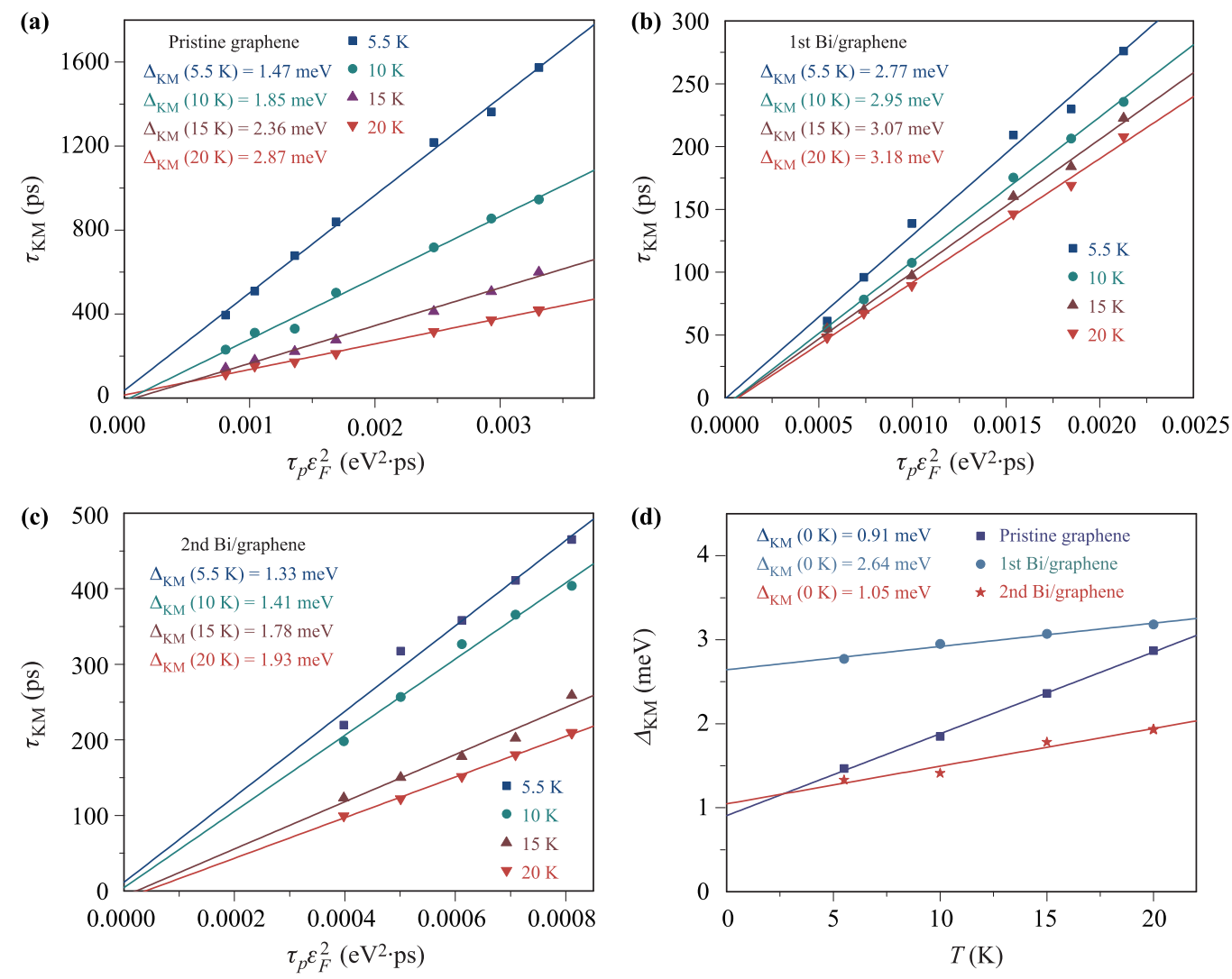

Fig. 4 Kane-Mele spin relaxation and its relationship with the SOC. $(\mathbf{a}-\mathbf{c}) \tau_{\mathrm{KM}}$ and $\tau_{p} \varepsilon_{F}^{2}$ data fitted using $\tau_{\mathrm{KM}}=\tau_{p}\left(\frac{\varepsilon_{F}}{\Delta}\right)^{2}$, yielding the SOC strength of $\mathrm{C} 0, \mathrm{C} 1$, and $\mathrm{C} 2$, respectively. (d) Kane-Mele-type SOC strength with respect to the temperature and the linear fits.

or the coverage of adatoms is often considered [7, 22, 41, 42]. After the first Bi-cluster deposition, the cluster coverage is low, as indicated by the $4.2 \%$ change in the resistance at room temperature. The interaction between the Bi clusters and vacancy defects may increase the SOC strength. For investigating the relationship between the SOC enhancement of the graphene and the Bi-cluster coverage, we deposited $\mathrm{Bi}$ clusters on the graphene device again. After the second Bi-cluster deposition $(\mathrm{C} 2)$, the overall metallic behavior was indicated by the $R-T$ curve, but the longitudinal resistance at room temperature increased by $163.4 \%$. This is attributed to excessive clusters inducing numerous scatterers, as shown in the AFM image. Figure 1(c) shows the surface morphology. The typical size of the Bi clusters was approximately $7 \mathrm{~nm}$. The areal cluster density was approximately $2 \times 10^{11} \mathrm{~cm}^{-2}$, and the distribution of the $\mathrm{Bi}$ clusters deposited on the graphene was not very uniform, owing to the fusion and growth of the clusters. The Bi clusters generated by a gas-aggregation cluster source were deposited on the graphene with a low kinetic energy. The deposition chamber was at room temperature, and the Bi clusters had enough energy to overcome the diffusion barrier. Thus, small clusters aggregated and coalesced into larger cluster [43]. The defects of the graphene sheet functioned as nucleation centers for the cluster growth, as described in the literature [44]. The density of hole carriers was lower than that of graphene after the first Bi-cluster deposition; thus, electrons were transferred from the Bi clusters to the graphene. Because of the high coverage of Bi-cluster scatterers, the carrier mobility decreased to $\sim 400 \mathrm{~cm}^{2} \cdot \mathrm{V}^{-1} \cdot \mathrm{s}^{-1}$. The WL is also observed in the MC, as shown in Fig. 2(c), where the WL is approximately half as strong as that of pristine graphene. Via the measurement of the WL at different gate voltages in Fig. 3(c), we obtained the spin-orbit interaction strength by using the aforementioned method. The extracted spin-relaxation time at different temperatures and back-gate voltages is shown in Fig. 4(c). The temperature-dependent SOC gap is shown in Fig. 4(d), indicating that at a high coverage of $\mathrm{Bi}$ clusters, the temperature-independent Kane-Mele SOC strength decreased to $1.05 \mathrm{meV}$, which is close to that for pristine graphene. The competition between defectcluster interaction and the fusion of clusters may explain this phenomenon. After the first Bi-cluster deposition, 
the clusters had low coverage, and small clusters interacted with the defects of the graphene, such as vacancies. However, after the second deposition, small clusters aggregated and coalesced into larger clusters [43]; thus, the interaction between the clusters and the defects of the graphene decreased, and the Kane-Mele SOC strength decreased. According to the slope of the linear fitting in Fig. 4(d), the temperature dependence of the SOC strength is similar in the graphene with low and high Bicluster coverage, because the cluster-induced scattering is dominant compared with the intrinsic scattering.

\section{Conclusion}

We investigated the effect of Bi clusters on WL and the spin-orbit interaction of a graphene sheet. While the charge concentration was kept constant, the amplitude of the MC correction was suppressed after the Bi-cluster deposition. Detailed WL data were obtained while the gate and temperature were changed. Fitting the WL data with E. McCann's formula yielded the spin-relaxation time for the graphene. The spin relaxation process followed Elliott-Yafet mechanism, originating from the intrinsic Kane-Mele SOC. The SOC strength was enhanced to $2.64 \mathrm{meV}$ by the first Bi-cluster deposition. The coverage of Bi clusters affected the resistance, carrier density, mobility, and WL of the graphene device.

Acknowledgements We gratefully acknowledge financial support from the National Key Projects for Basic Research of China (Grant No. 2013CB922103), the National Natural Science Foundation of China (Grant Nos. 91421109, 91622115, 11522432, 61176088, and 11274003), the Natural Science Foundation of Jiangsu Province (Grant Nos. BK20130054 and BK20160659), and the Fundamental Research Funds for the Central Universities. The opening Project of Wuhan National High Magnetic Field Center, supported the pulsed magnetic field.

Open Access This article is distributed under the terms of the Creative Commons Attribution License which permits any use, distribution, and reproduction in any medium, provided the original author(s) and the source are credited.

\section{References}

1. C. L. Kane and E. J. Mele, Quantum spin Hall effect in graphene, Phys. Rev. Lett. 95(22), 226801 (2005)

2. J. Zhang, C. Triola, and E. Rossi, Proximity effect in graphene-topological-insulator heterostructures, Phys. Rev. Lett. 112(9), 096802 (2014)

3. K. H. Jin and S. H. Jhi, Proximity-induced giant spinorbit interaction in epitaxial graphene on a topological insulator, Phys. Rev. B 87(7), 075442 (2013)
4. Z. Qiao, W. Ren, H. Chen, L. Bellaiche, Z. Zhang, A. H. MacDonald, and Q. Niu, Quantum anomalous Hall effect in graphene proximity coupled to an antiferromagnetic insulator, Phys. Rev. Lett. 112(11), 116404 (2014)

5. J. Zhou, Q. Liang, and J. Dong, Enhanced spin-orbit coupling in hydrogenated and fluorinated graphene, Carbon 48(5), 1405 (2010)

6. M. Gmitra, D. Kochan, and J. Fabian, Spin-orbit coupling in hydrogenated graphene, Phys. Rev. Lett. 110(24), 246602 (2013)

7. A. Cresti, D. Van Tuan, D. Soriano, A. W. Cummings, and S. Roche, Multiple quantum phases in graphene with enhanced spin-orbit coupling: From the quantum spin Hall regime to the spin Hall effect and a robust metallic state, Phys. Rev. Lett. 113(24), 246603 (2014)

8. C. Weeks, J. Hu, J. Alicea, M. Franz, and R. Wu, Engineering a robust quantum spin Hall state in graphene via adatom deposition, Phys. Rev. X 1(2), 021001 (2011)

9. A. H. Castro Neto and F. Guinea, Impurity-induced spin-orbit coupling in graphene, Phys. Rev. Lett. 103(2), 026804 (2009)

10. H. Jiang, Z. Qiao, H. Liu, J. Shi, and Q. Niu, Stabilizing topological phases in graphene via random adsorption, Phys. Rev. Lett. 109(11), 116803 (2012)

11. P. Lee, K. H. Jin, S. J. Sung, J. G. Kim, M. T. Ryu, H. M. Park, S. H. Jhi, N. Kim, Y. Kim, S. U. Yu, K. S. Kim, D. Y. Noh, and J. Chung, Proximity effect induced electronic properties of graphene on $\mathrm{Bi}_{2} \mathrm{Te}_{2} \mathrm{Se}$, ACS Nano 9(11), 10861 (2015)

12. S. Rajput, Y. Y. Li, M. Weinert, and L. Li, Indirect interlayer bonding in graphene-topological insulator van der Waals heterostructure: Giant spin-orbit splitting of the graphene Dirac states, ACS Nano 10(9), 8450 (2016)

13. J. Balakrishnan, G. Kok Wai Koon, M. Jaiswal, A. H. Castro Neto, and B. Ozyilmaz, Colossal enhancement of spin-orbit coupling in weakly hydrogenated graphene, Nat. Phys. 9(5), 284 (2013)

14. J. Balakrishnan, G. K. W. Koon, A. Avsar, Y. Ho, J. H. Lee, M. Jaiswal, S. J. Baeck, J. H. Ahn, A. Ferreira, M. A. Cazalilla, A. H. C. Neto, and B. Özyilmaz, Giant spin Hall effect in graphene grown by chemical vapour deposition, Nat. Commun. 5, 4748 (2014)

15. Y. Han, G. X. Ge, J. G. Wan, J. J. Zhao, F. Q. Song, and G. H. Wang, Predicted giant magnetic anisotropy energy of highly stable Ir dimer on single-vacancy graphene, Phys. Rev. B 87(15), 155408 (2013)

16. M. V. Ulybyshev and M. I. Katsnelson, Magnetism and interaction-induced gap opening in graphene with vacancies or hydrogen adatoms: Quantum Monte Carlo study, Phys. Rev. Lett. 114(24), 246801 (2015)

17. Y. C. Lin, P. Y. Teng, P. W. Chiu, and K. Suenaga, Exploring the single atom spin state by electron spectroscopy, Phys. Rev. Lett. 115(20), 206803 (2015) 
18. X. Li, Y. Zhu, W. Cai, M. Borysiak, B. Han, D. Chen, R. D. Piner, L. Colombo, and R. S. Ruoff, Transfer of largearea graphene films for high-performance transparent conductive electrodes, Nano Lett. 9(12), 4359 (2009)

19. M. Han, C. Xu, D. Zhu, L. Yang, J. Zhang, Y. Chen, K. Ding, F. Song, and G. Wang, Controllable synthesis of two-dimensional metal nanoparticle arrays with oriented size and number density gradients, Adv. Mater. 19(19), 2979 (2007)

20. F. Song, M. Han, M. Liu, B. Chen, J. Wan, and G. Wang, Experimental observation of nanojets formed by heating PbO-coated Pb clusters, Phys. Rev. Lett. 94(9), 093401 (2005)

21. Y. W. Tan, Y. Zhang, H. L. Stormer, and P. Kim, Temperature dependent electron transport in graphene, Eur. Phys. J. Spec. Top. 148(1), 15 (2007)

22. J. H. Chen, C. Jang, S. Adam, M. S. Fuhrer, E. D. Williams, and M. Ishigami, Charged-impurity scattering in graphene, Nat. Phys. 4(5), 377 (2008)

23. Z. Jia, B. Yan, J. Niu, Q. Han, R. Zhu, D. Yu, and X. $\mathrm{Wu}$, Transport study of graphene adsorbed with indium adatoms, Phys. Rev. B 91(8), 085411 (2015)

24. K. Pi, W. Han, K. M. McCreary, A. G. Swartz, Y. Li, and R. K. Kawakami, Manipulation of spin transport in graphene by surface chemical doping, Phys. Rev. Lett. 104(18), 187201 (2010)

25. X. Wang, X. Pan, M. Gao, J. Yu, J. Jiang, J. Zhang, H. Zuo, M. Zhang, Z. Wei, W. Niu, Z. Xia, X. Wan, Y. Chen, F. Song, Y. Xu, B. Wang, G. Wang, and R. Zhang, Evidence of both surface and bulk Dirac bands and anisotropic nonsaturating magnetoresistance in ZrSiS, Adv. Electron. Mater 2 (10) (2016)

26. E. McCann and V. I. Fal'ko, $z \rightarrow-z$ symmetry of spinorbit coupling and weak localization in graphene, Phys. Rev. Lett. 108(16), 166606 (2012)

27. E. McCann and V. I. Fal'ko, Weak localization and spinorbit coupling in monolayer and bilayer graphene, in: $\mathrm{H}$. Aoki and S. M. Dresselhaus (Eds.), Physics of Graphene, Springer International Publishing, 2014

28. F. V. Tikhonenko, A. A. Kozikov, A. K. Savchenko, and R. V. Gorbachev, Transition between electron localization and antilocalization in graphene, Phys. Rev. Lett. 103(22), 226801 (2009)

29. K. I. Imura, Y. Kuramoto, and K. Nomura, Weak localization properties of the doped $Z_{2}$ topological insulator, Phys. Rev. B 80(8), 085119 (2009)

30. K. I. Imura, Y. Kuramoto, and K. Nomura, Antilocalization of graphene under the substrate electric field, Europhys. Lett. 89(1), 17009 (2010)
31. H. Min, J. E. Hill, N. A. Sinitsyn, B. R. Sahu, L. Kleinman, and A. H. MacDonald, Intrinsic and Rashba spin-orbit interactions in graphene sheets, Phys. Rev. B 74(16), 165310 (2006)

32. M. Zarea and N. Sandler, Rashba spin-orbit interaction in graphene and zigzag nanoribbons, Phys. Rev. B 79(16), 165442 (2009)

33. P. Rakyta, A. Kormányos, and J. Cserti, Trigonal warping and anisotropic band splitting in monolayer graphene due to Rashba spin-orbit coupling, Phys. Rev. B 82(11), 113405 (2010)

34. D. Huertas-Hernando, F. Guinea, and A. Brataas, Spinorbit-mediated spin relaxation in graphene, Phys. Rev. Lett. 103(14), 146801 (2009)

35. E. V. Zhizhin, A. Varykhalov, A. G. Rybkin, A. A. Rybkina, D. A. Pudikov, D. Marchenko, J. SánchezBarriga, I. I. Klimovskikh, G. G. Vladimirov, O. Rader, and A. M. Shikin, Spin splitting of Dirac fermions in graphene on $\mathrm{Ni}$ intercalated with alloy of $\mathrm{Bi}$ and $\mathrm{Au}$, Carbon 93, 984 (2015)

36. S. V. Morozov, K. S. Novoselov, M. I. Katsnelson, F. Schedin, L. A. Ponomarenko, D. Jiang, and A. K. Geim, Strong suppression of weak localization in graphene, Phys. Rev. Lett. 97(1), 016801 (2006)

37. F. V. Tikhonenko, D. W. Horsell, R. V. Gorbachev, and A. K. Savchenko, Weak localization in graphene flakes, Phys. Rev. Lett. 100(5), 056802 (2008)

38. X. Wu, X. Li, Z. Song, C. Berger, and W. A. de Heer, Weak antilocalization in epitaxial graphene: Evidence for chiral electrons, Phys. Rev. Lett. 98(13), 136801 (2007)

39. G. Fishman and G. Lampel, Spin relaxation of photoelectrons in p-type gallium arsenide, Phys. Rev. B 16(2), 820 (1977)

40. Y. Ohno, R. Terauchi, T. Adachi, F. Matsukura, and H. Ohno, Spin relaxation in GaAs(110) quantum wells, Phys. Rev. Lett. 83(20), 4196 (1999)

41. A. Ferreira, T. G. Rappoport, M. A. Cazalilla, and A. H. Castro Neto, Extrinsic spin Hall effect induced by resonant Skew scattering in graphene, Phys. Rev. Lett. 112(6), 066601 (2014)

42. J. Hu, J. Alicea, R. Wu, and M. Franz, Giant topological insulator gap in graphene with 5 d adatoms, Phys. Rev. Lett. 109(26), 266801 (2012)

43. J. M. Zuo and B. Q. Li, Nanostructure evolution during cluster growth: Ag on H-terminated $\mathrm{Si}(111)$ surfaces, Phys. Rev. Lett. 88(25), 255502 (2002)

44. F. J. Ribeiro, J. B. Neaton, S. G. Louie, and M. L. Cohen, Mechanism for bias-assisted indium mass transport on carbon nanotube surfaces, Phys. Rev. B 72(7), $075302(2005)$ 